\title{
EDITORIAL
}

\section{Poor-quality prenatal dietary patterns are related to the mental health of mothers and children - could dietary improvement break the cycle?}

\author{
Samantha L. Dawson, ${ }^{1,2}$ Wolfgang Marx, ${ }^{1,3}$ Felice Jacka ${ }^{1,4,5}$ \\ ${ }^{1}$ Food and Mood Centre, IMPACT SRC School of Medicine, Deakin University, Geelong, VIC, Australia. ${ }^{2}$ Early Life Epigenetics Group, \\ Murdoch Children's Research Institute, Royal Children's Hospital, Parkville, VIC, Australia. ${ }^{3}$ School of Allied Health, La Trobe University, \\ Bundoora, VIC, Australia. ${ }^{4}$ Centre for Adolescent Health, Murdoch Children's Research Institute, Royal Children's Hospital, Parkville, VIC, \\ Australia. ${ }^{5}$ Black Dog Institute, Randwick, NSW, Australia.
}

Poor-quality prenatal diets are related to antenatal depressive symptoms and stress in women. ${ }^{1}$ Likewise, prenatal depressive symptoms and unhealthy diets are both independently associated with higher levels of child emotional-behavioral dysregulation. ${ }^{2,3}$ Addressing diet may influence multiple interconnected pathways that are relevant to the pathogenesis of mental disorder, such as gut microbiota, inflammatory status, the hypothalamicpituitary-adrenal axis, oxidative stress, and brain-derived neurotrophic factors. This raises the question: can dietary improvement in pregnancy prevent mental disorders in mothers and help prevent the intergenerational transmission of mental ill-health to children? If so, what dietary advice should be followed, and would this advice change based on the disorder?

To inform these decisions, we look to studies such as Paskulin et al., where in 712 southern Brazilian women, low intakes of healthy foods and high intakes of refined sugars during pregnancy were related to an increased prevalence of maternal mental disorder. ${ }^{4}$ Importantly, major depressive disorder and generalized anxiety were examined as separate outcomes; this is useful because major depressive disorder and generalized anxiety are highly comorbid, and previous studies examining the association between maternal diet and generalized anxiety showed inconsistent results. ${ }^{1}$

In this context, Paskulin et al. reported that dietary patterns were related to the prevalence of major depressive disorder in pregnant women, but were not related to generalized anxiety. ${ }^{4}$ Women consuming a standard Brazilian diet (such as rice or noodles, French rolls, coffee with sugar, margarine, and artificial juices) had a higher probability of major depressive disorder than those consuming a varied diet. When food groups were analysed in comparison to the recommended intakes, major depressive disorder prevalence was higher for women

Correspondence: Samantha Dawson, Food \& Mood Centre, School of Medicine, Deakin University, PO Box 281, 3220, Geelong, Victoria, Australia.

E-mail: samantha.dawson@ deakin.edu.au

Epub Aug 312017. with a low intake of fruit and a high intake of sugars, while generalized anxiety disorder prevalence was higher for women with a low intake of beans. While acknowledging the potential for this to be a chance finding, it is tempting to speculate that the association between a lower bean intake and a higher prevalence ratio of anxiety disorder, as reported by Paskulin et al., may be at least in part due to functional differences in the gut microbiota arising from a limited intake of fermentable fiber found in beans. Fermentable fiber influences gut microbiota composition and the production of short chain fatty acids; these, in turn, have been demonstrated to affect mood in animal models, as well as influence neurotransmitters such as serotonin. ${ }^{5}$

Observational studies consistently associate poor prenatal diet quality with poorer maternal and offspring mental health outcomes. ${ }^{1-3}$ Prenatal diet quality thus presents a potentially important and modifiable target for reducing the risk of mental disorders in mothers and offspring alike. As the potential of dietary interventions as a treatment for mental illness is relatively understudied, large clinical trials are required to confirm causality. One recent randomized controlled trial reported that a modified Mediterranean diet reduced depression symptoms in adults compared to a control group. ${ }^{6}$ While these results are promising, they are considered preliminary and require replication in larger samples. Such trials should specifically target diet quality by increasing the consumption of fresh plants and fibrous whole grains and legumes, non-processed meats and fish, and healthy oils, and reducing refined high-fat and sugar-rich foods. Future studies should also evaluate the underlying mechanisms by measuring parameters associated with the relevant biological pathways for various mental disorders. If efficacious, dietary modification strategies could provide a low-cost, safe, and simple strategy for improving mental health and be a useful step towards breaking the cycle of intergenerational transmission of mental disorders.

\section{Disclosure}

The authors report no conflicts of interest. 


\section{References}

1 Baskin R, Hill B, Jacka FN, O'Neil A, Skouteris H. The association between diet quality and mental health during the perinatal period. A systematic review. Appetite. 2015;91:41-7.

2 Jacka FN, Ystrom E, Brantsaeter AL, Karevold E, Roth C, Haugen M, et al. Maternal and early postnatal nutrition and mental health of offspring by age 5 years: a prospective cohort study. J Am Acad Child Adolesc Psychiatry. 2013;52:1038-47.

3 Pina-Camacho L, Jensen SK, Gaysina D, Barker ED. Maternal depression symptoms, unhealthy diet and child emotional-behavioural dysregulation. Psychol Med. 2015;45:1851-60.
4 Paskulin JT, Drehmer M, Olinto MT, Hoffmann JF, Pinheiro AP, Schmidt $\mathrm{Ml}$, et al. Association between dietary patterns and mental disorders in pregnant women in Southern Brazil. Rev Bras Psiquiatr. 2017;39:208-15.

5 Dash S, Clarke G, Berk M, Jacka FN. The gut microbiome and diet in psychiatry: focus on depression. Curr Opin Psychiatry. 2015;28: 1-6.

6 Jacka FN, O'Neil A, Opie R, Itsiopoulos C, Cotton S, Mohebbi M, et al. A randomised controlled trial of dietary improvement for adults with major depression (the 'SMILES' trial). BMC Med. 2017;15:23. 